\title{
A Dynamic Model of a Passive Magnetic Attitude Control System for the RAX Nanosatellite*
}

\author{
G. Park* and S. Seagraves* \\ Department of Aerospace Engineering, University of Michigan, Ann Arbor, MI, 48109, USA \\ N. H. McClamroch ${ }^{\dagger}$ \\ ${ }^{\ddagger}$ Department of Aerospace Engineering, University of Michigan, Ann Arbor, MI, 48109, USA
}

The Radio Aurora Explorer (RAX) is an NSF sponsored nanosatellite being designed, built, and tested by students at the University of Michigan; it is currently scheduled for launch in 2010. The spacecraft utilizes a passive magnetic attitude control system consisting of a permanent magnet aligned with the axis of symmetry of the nanosatellite, and two magnetic hysteresis rods, each aligned along the other two principal axes of the nanosatellite; these hysteresis rods provide energy dissipation necessary to achieve alignment of the axis of the symmetry with the Earth's magnetic field. This paper presents an analytical model for the passive magnetically controlled attitude dynamics of the RAX nanosatellite, along with numerical simulations to assess the properties of the attitude dynamics.

\section{Nomenclature}

\begin{tabular}{|c|c|}
\hline$R_{I}^{B}$ & $3 \times 3$ rotation (or transformation) matrix from the inertial frame to the body-fixed frame \\
\hline & $3 \times 3$ rotation (or transformation) matrix from the inertial frame to the Earth-fixed frame \\
\hline & $3 \times 3$ rotation (or transformation) matrix from the Earth-fixed frame to the body-fixed frame \\
\hline$b_{1}, b_{2}, b_{3}$ & unit vectors that define the body-fixed frame as RAX principal axes; the longitudinal axes \\
\hline & $\begin{array}{l}\text { of the two hysteresis rods are aligned with the first two principal axes, the longitudinal axis from south } \\
\text { pole to north pole of the permanent magnet is aligned along the third principal axis }\end{array}$ \\
\hline$\omega$ & angular velocity of the spacecraft expressed in the body-fixed frame $(\mathrm{rad} / \mathrm{s})$ \\
\hline$\omega_{0}$ & scalar rotation rate of the Earth about its north-south axis $(\mathrm{rad} / \mathrm{s})$ \\
\hline$M$ & magnetic torque vector in the body-fixed frame $(N-m)$ \\
\hline$J$ & moment of inertia of the RAX nanosatellite expressed in the body-fixed frame $\left(k g-m^{2}\right)$ \\
\hline$B_{E}$ & Earth magnetic field (flux density) vector at the orbital location of the RAX in Earth-fixed frame (Teslas) \\
\hline$H_{E}$ & Earth magnetic field strength vector at the orbital location of the RAX in the Earth-fixed frame $(A / m)$ \\
\hline$\mu_{0}$ & constant permeability of vacuum $\left(N / A^{2}\right)$ \\
\hline$B_{p}$ & constant magnetic field (flux density) in the permanent magnet (Teslas) \\
\hline$V_{p}$ & volume of permanent magnet $\left(\mathrm{m}^{3}\right)$ \\
\hline$H^{i}$ & component of the Earth's magnetic field strength along the longitudinal axis of the ith hysteresis rod $(A / m)$ \\
\hline$B^{i}$ & magnetic field (flux density) induced in the ith hysteresis rod by the Earth's magnetic field (Teslas) \\
\hline$H_{c}$ & constant characterizing the coercivity value of the hysteresis major loop for each of the hysteresis rods $(A / m)$ \\
\hline$H_{r}$ & constant characterizing the remanence value of the hysteresis major loop for each of the hysteresis rods $(A / m)$ \\
\hline$B_{m}$ & constant saturation value characterizing the hysteresis major loop for each of the hysteresis rods (Teslas) \\
\hline$V_{h}$ & volume of each hysteresis rod $\left(m^{3}\right)$ \\
\hline
\end{tabular}

\footnotetext{
*Graduate student, Aerospace Engineering, University of Michigan, and AIAA Member.

${ }^{\dagger}$ Professor, Aerospace Engineering, University of Michigan, and AIAA Member.

$\ddagger *$ This research has been supported in part by NSF under grant CMS-0555797.
} 


\section{Introduction}

\section{A. Motivation}

A passive magnetic attitude control system does not involve any components with moving parts. There Aare no external energy requirements, and additional sensors or actuators, beyond a permanent magnet and hysteresis rods, are not required. Such attitude control systems are inexpensive and reliable, which is their main attraction. On the other hand, they are not programmable and they have limited capability for attitude stabilization.

While passive magnetic attitude control has been utilized in past small satellite missions, the attitude performance of such satellites remains somewhat mysterious. Complete analytical models of the relevant three dimensional attitude dynamics and kinematics seem not to have been developed. The intent of this paper is to develop a fully descriptive and accurate attitude dynamics model for the RAX nanosatellite that incorporates a passive magnetic attitude control system consisting of a permanent magnet and two hysteresis rods that are rigidly attached to the RAX; this model can also be adapted to apply to the attitude dynamics of other spacecraft controlled by a passive magnetic system. In addition to the analytical model, several theoretical results are presented for the idealized assumption that the magnitude and direction of the Earth's magnetic field are constant along the RAX orbit. Numerical simulations are also presented, assessing the accuracy of the passive magnetic control approach for the RAX nanosatellite.

The NSF sponsored Radio Aurora Explorer (RAX) project currently underway at the University of Michi$\operatorname{gan}^{16}$ provides a unique opportunity and motivation to develop an analytical model of the passive magnetically controlled nanosatellite attitude dynamics and to produce simulations and predictions that can, in the present, be used to confirm design features and, in the future, be validated with actual mission data.

\section{B. RAX Mission Overview}

The RAX nanosatellite has been commissioned to study irregularities in the Earth's electron density in the polar ionosphere. The spacecraft is being designed and built to the CubeSat standard developed by engineers at California Polytechnic State University. RAX is a triple configuration (3U) CubeSat that will utilize Cal Poly's standardized P-POD deployment system. Upon release from the P-POD, the RAX nanosatellite enters a circular orbit with random orientation and angular velocity due to uncertainties in deployment. The passive magnetic attitude stabilization system is designed to slow any tumbling of the RAX nanosatellite due to deployment and to align the nanosatellite's primary principal axis, namely its third principal axis which is also the longitudinal axis of the permanent magnet on the RAX, with the local direction of the Earth's magnetic field.

A schematic of the RAX nanosatellite is shown in Figure 1. It is essentially a box shaped spacecraft, with an axis of symmetry along its long axis. Three body-fixed principal axes, with origin located at the center of mass of RAX, are shown.

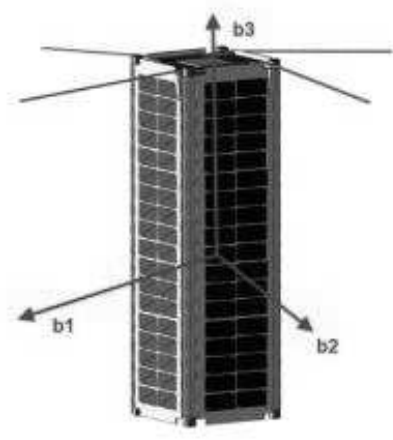

Figure 1. Schematic of the RAX nanosatellite 


\section{Contributions of the paper}

Achieving the attitude stabilization objectives is necessary for success of the RAX mission. Although prior spacecraft missions, using similar magnetic control systems, have been successful, many questions remain about design of such magnetic control systems and the resulting attitude dynamics performance. A careful analysis of such magnetic attitude control systems is complicated by the facts that the RAX can undergo very complex rotational motion, the hysteresis rods provide low-authority nonlinear energy dissipation, and the variation of the magnitude and direction of the Earth's magnetic field along the RAX orbit can be extremely irregular.

The RAX attitude model, models for the permanent magnet and the hysteresis rods, and models of the Earth's magnetic field along the RAX orbit are developed in an integrated form. This complete model of the passive magnetic attitude control system for the RAX is an important contribution of this paper. Globally valid attitude models are developed to describe complex spacecraft rotations including tumbling; these models are constructed using frame transformation or rotation matrices that are globally defined without singularities or ambiguities that arise from use of Euler angles or quaternions.

In addition, detailed models of the hysteresis rods are expressed in a complete and unambiguous form; these models for the torques on the RAX generated by the hysteresis rods are consistent with the physics of hysteresis materials. A novel feature is that the hysteresis models are physically valid only within certain regions of operation.

Formal methods of analysis are used to summarize (without proof) certain attitude performance characteristics that hold for the special case that the magnitude and direction of the Earth's magnetic field are constant; this is not a realistic assumption for the RAX nanosatellite but it provides insight into the realistic case that the magnitude and direction of the Earth's magnetic field change slowly. For the more general case, the integrated model of the RAX magnetic attitude control system is used as a basis for simulation studies.

The simulation studies are based on integration algorithms that are especially suited for the RAX attitude dynamics. The low-authority of the hysteresis rods suggests that commonly used numerical integration schemes (such as MATLAB's ODE45 based on standard Runge-Kutta integration algorithms) could generate significant numerical errors, comparable to the dissipation effects from the hysteresis rods; such a numerical approach would not be satisfactory. Recently, a Lie group variational integrator (LGVI) has been developed at the University of Michigan; this numerical integration algorithm for rigid body attitude dynamics substantially reduces the accumulation of such numerical dissipation errors and it has other accuracy and efficiency properties. This new LGVI algorithm has been incorporated into the numerical simulations presented in this paper providing much more accurate simulation results over the necessarily extended time spans of interest for the RAX attitude dynamics.

The development in this paper can be compared with an integrated models of the attitude dynamics of an orbiting nano-satellite, as described in a recent AIAA paper. ${ }^{17}$ This paper includes simulation models for the orbit parameters, gravity gradient torque, aerodynamic torque, and magnetic torques. The results and conclusions are in general agreement with those in the present paper. The present paper provides a more complete description of the various reference frames, hysteresis models, and the numerical integration scheme.

\section{Attitude Dynamics Model}

\section{A. Simplifications and Assumptions}

For this preliminary investigation, we consider a simplified model; however, the simplifications may be replaced by more complex and realistic models as future investigations progress. We assume the RAX nanosatellite is in a circular orbit at the mission-specified altitude of $650 \mathrm{~km}$ and an orbit inclination angle of $72^{\circ}$. We consider the spacecraft to be an axially-symmetric rigid body, with known principal axes. We utilize simplified models of the Earth's magnetic field and of the torques generated by the permanent magnet 
and by the two hysteresis rods. To represent the Earth's magnetic field, we make use of the publicly available International Geomagnetic Reference Field (IGRF). ${ }^{13}$ This model provides numerical values for the magnetic field vector at each point along the RAX orbit. Based on the Earth's magnetic field at a given point on the orbit, the magnetic torques due to the permanent magnet and the two hysteresis rods are modeled using standard theory. Modeling the torques due to the hysteresis rods is complicated; many of the models of hysteresis rods proposed in the literature are incomplete. One of the contributions of this paper is to provide a logically correct and physically consistent model of the energy dissipation due to the hysteresis rods; our model is based on prior developments in. ${ }^{2,5}$ We provide a detailed hysteresis model that takes into account the magnetic characteristics of the hysteresis rods, including hysteresis effects, saturation effects, and the limited region of physical validity of the hysteresis model. These models are presented for arbitrary rotational motion of the RAX nanosatellite and for arbitrary direction of the Earth's magnetic field at RAX's location on its orbit.

\section{B. Reference Frame Definitions}

In order to accurately represent the attitude of the RAX nanosatellite, several different reference frames are contructed.

The Earth-centered inertial frame has its origin at the center of the Earth and remains fixed with respect to the Earth's rotation. The axes are denoted by X,Y,Z and are defined as follows:

- The Z-axis points due north along the Earth's axis of rotation

- The X-axis points in the direction of the Vernal Equinox

- The Y-axis completes the right-hand frame

The Earth-centered Earth-fixed frame also has its origin at the center of the Earth; however this frame rotates with the Earth. Thus the coordinates of a point fixed to the surface of the Earth do not change. These axes are denoted $X^{\prime}, Y^{\prime}, Z^{\prime}$ and are defined as follows:

- The $Z^{\prime}$-axis points due north along the Earth's axis of rotation

- The $X^{\prime}$-axis intersects the surface of the Earth at $0^{\circ}$ latitude, $0^{\circ}$ longitude

- The $Y^{\prime}$-axis completes the right-hand frame

The RAX body-fixed frame has its origin at the RAX's center of mass. Unit vectors that define the spacecraft principal axes are denoted $b_{1}, b_{2}, b_{3}$ and are defined as follows:

- $b_{1}$ and $b_{2}$ point outward normal to the side panels of RAX

- $b_{3}$ points outward normal to the top panel, along the axis of symmetry of RAX

\section{Rotation Matrices}

In order to carry out transformations between these reference frames, rotation matrices ${ }^{3}$ are utilized. The rotation matrix from the inertial to the Earth-fixed frame is denoted as $R_{I}^{E}$, the rotation matrix from the Earth-fixed to the body-fixed frame is denoted as $R_{E}^{B}$, the rotation matrix from the inertial frame to the body-fixed frame is denoted as $R_{I}^{B}$.

The following relationship involving these three rotation matrices holds:

$$
R_{E}^{B} R_{I}^{E}=R_{I}^{B}
$$

The rotation matrix $R_{I}^{E}$ from the inertial frame to the Earth-fixed frame is the time-varying marix function

$$
R_{I}^{E}=\left[\begin{array}{ccc}
\cos \omega_{0} t & -\sin \omega_{0} t & 0 \\
\sin \omega_{0} t & \cos \omega_{0} t & 0 \\
0 & 0 & 1
\end{array}\right]
$$




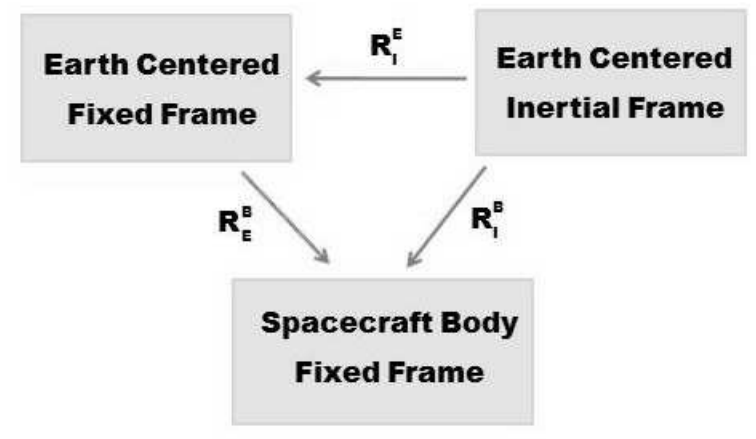

Figure 2. The relationship between the three rotation matrices

where $\omega_{0}$ is the constant rotation rate of the Earth about its north-south axis. This assumes that at the initial time, taken to be time zero, the Earth-fixed axes are exactly aligned with the inertial axes.

The attitude kinematics and the attitude dynamics equations are expressed in terms of the rotation matrix from the inertial frame to the body-fixed frame $R_{I}^{B}$ and the angular velocity vector $\omega$ expressed in the body-fixed frame. This rotation matrix is also viewed as defining the attitude of the RAX nanosatellite, by providing the relationship between each body fixed axis and each inertial axis.

The attitude dynamics equations express the rotational acceleration of the RAX, in the body fixed frame, in terms of the external torques that act on the RAX. In the subsequent analysis, the only external torques are due to the magnetic torques on the RAX that arise from the permanent magnet and the two hysteresis rods. Gravity gradient torques, torques due to solar radiation effects, and other external torque sources are ignored in the present development.

\section{Kinematics and Dynamics}

In mathematical terms, the rotational kinematics and rotational dynamics are described by the equations ${ }^{3}$

$$
\begin{aligned}
& \dot{R}_{I}^{B}+S(\omega) R_{I}^{B}=0 \\
& J \dot{\omega}+\omega \times J \omega=M
\end{aligned}
$$

where $J$ is the RAX moment of inertia matrix and $M$ is the total magnetic torque on the RAX expressed in the body-fixed frame. The skew-symmetric matrix is

$$
S(\omega)=\left[\begin{array}{ccc}
0 & \omega_{3} & -\omega_{2} \\
-\omega_{3} & 0 & \omega_{1} \\
\omega_{2} & -\omega_{1} & 0
\end{array}\right]
$$

The RAX nanosatellite has an axis of symmetry which is assumed to be its third principal axis. This implies that the moment of inertia matrix is a diagonal matrix with first two entries on the diagonal equal. The axial symmetry of the RAX is a special feature that is not important in the subsequent development; that is, the subsequent development is valid for an asymmetric satellite.

\section{E. Magnetic Torques on the RAX}

Magnetic stabilization of the RAX attitude motion is based on the interaction between the Earth's magnetic field and the torques generated by the magnetic materials within the RAX structure. A permanent magnet is embedded in RAX with its longitudinal axis from south pole to north pole aligned with the RAX third principal axis. In addition, two hysteresis rods are embedded in the RAX nanosatellite, one with its longitudinal axis aligned along the RAX first principal axis and the other with its longitudinal axis aligned along 
the RAX second principal axis. Thus the longitudinal axes of the permanent magnet and the two hysteresis rods are mutually orthogonal.

\section{Permanent Magnet Torque}

The Earth's magnetic field vector at the location of the RAX nanosatellite in its orbit is obtained from a standard Earth magnetic field model. The magnetic field flux density vector in the Earth-fixed frame is denoted as $B_{E}$. Note that this depends on properties of the RAX orbit, namely the orbital radius and orbit inclination angle. The magnetic field strength vector of the Earth's magnetic field, denoted by $H_{E}$ is related to the magnetic field flux density $B_{E}$ of the Earth according to

$$
H_{E}=\frac{B_{E}}{\mu_{0}} .
$$

where $\mu_{0}$ is the permeability of the vacuum in space.

The constant strength of the magnetic dipole produced by the permanent magnet is denoted by $B_{p}$; it can be calculated directly from properties of the magnetic material. The longitudinal axis of the permanent magnet is aligned with the third principal axis of the RAX nanosatellite, in the sense that the direction of the longitudinal axis of the permanent magnet, from south pole to north pole, is $b_{3}$. The magnetic torque vector generated by the interaction of the permanent magnet and the Earth's magnetic field is given by

$$
M_{p}=B_{p} V_{p} b_{3} \times R_{E}^{B} H_{E} .
$$

Here $V_{p}$ is the volume of the permanent magnet. The direction of the torque produced by the permanent magnet is orthogonal to the longitudinal axis of the permanent magnet and to the direction of the Earth's magnetic field at the RAX location.

\section{Torques due to Hysteresis Rods}

Each of the hysteresis rods produces a magnetic dipole which is not constant, but is proportional to the component of the Earth's magnetic field along the longitudinal axis of that hysteresis rod. Hysteresis magnetic materials are much like permanent magnets in their function, except that they are of significantly higher permeability. This means that under the influence of a changing external magnetic field (such as the Earth's), the hysteresis materials tend to exhibit a realignment of micro-magnetic dipoles and a change in magnetic domain boundaries. These changes result in frictional dissipation of energy at the molecular level, a phenomenon known as hysteresis dissipation. ${ }^{12}$ This realignment is dynamic in the sense that the magnetic interaction between the hysteresis rod and the external field depends upon memory in the material.

Various mathematical models for hysteresis rods have been proposed in the literature; ${ }^{4,9}$ most of these models are incomplete in the sense that they are only described for the case of a simple sinusoidal time variation in the Earth's magnetic strength in the direction of the hysteresis rod. Such models are physically incomplete in that they do not fully describe the hysteresis memory, and they are incomplete in the sense that they are not mathematically well-posed.

Our development follows the model of hysteresis rods given in $;{ }^{2,5}$ we provide a general dynamic description of each hysteresis rod in terms of a single ordinary differential equation that depends on several physical parameters that describe the magnetic properties of the hysteresis rod materials. The differential equation for each hysteresis rod clearly identifies the memory, namely the induced magnetic flux density in the hysteresis rod. In addition, there are physical limits associated with the hysteresis model; these limits are explained and incorporated into the attitude dynamics equations for the RAX. The subsequent development assumes that the two hysteresis rods are physically identical.

The component of the Earth's magnetic field in the direction of hysteresis rod $i$ is

$$
H^{i}=b_{i}^{T} R_{E}^{B} H_{E}, i=1,2 .
$$


Based on the presentation in $^{2,5}$ the induced magnetic flux density in hysteresis rod $i$ is described by the differential equation: if $\dot{H}^{i} \geq 0$, then

$$
\dot{B^{i}}=\frac{2 B_{m}}{H_{r} \pi}\left(\frac{\left(H^{i}+H_{c}\right) \cos \left(\frac{\pi B^{i}}{2 B_{m}}\right)-H_{r} \sin \left(\frac{\pi B^{i}}{2 B_{m}}\right)}{2 H_{c}}\right)^{2} \dot{H}^{i},
$$

while if $\dot{H}^{i}<0$, then the differential equation is

$$
\dot{B^{i}}=\frac{2 B_{m}}{H_{r} \pi}\left(\frac{\left(H_{c}-H^{i}\right) \cos \left(\frac{\pi B^{i}}{2 B_{m}}\right)+H_{r} \sin \left(\frac{\pi B^{i}}{2 B_{m}}\right)}{2 H_{c}}\right)^{2} \dot{H}^{i},
$$

Here $B_{m}$ is the constant saturation value of the induced magnetic flux density of the hysteresis rod, $H_{c}$ is a constant coercivity value of the hysteresis material, and $H_{r}$ is a constant remanence value of the hysteresis material.

The above hysteresis model is physically meaningful for each hysteresis rod only if the following two inequalities are satisfied:

$$
-H_{c} \leq H^{i}-H_{r} \tan \left(\frac{\pi B^{i}}{2 B_{m}}\right) \leq+H_{c}, i=1,2 .
$$

It can be shown that if the initial conditions $B^{i}(0)$ and $H^{i}(0)$ satisfy the above hysteresis inequalities, then the inequalities are always satisfied. That is, the region defined by the above two inequalities is invariant.

The boundaries of this hysteresis region are obtained by setting the above inequalities to equalities. These conditions for equality define two "S-shaped" curves in the $B^{i}$ versus $H^{i}$ plane. The interior of these two curves, where both inequalities are satisfied, is the hysteresis region. These two boundary curves given by

$$
H^{i}-H_{r} \tan \left(\frac{\pi B^{i}}{2 B_{m}}\right)=+H_{c}
$$

for increasing $H^{i}$ and by

$$
H^{i}-H_{r} \tan \left(\frac{\pi B^{i}}{2 B_{m}}\right)=-H_{c}
$$

for decreasing $H^{i}$ define the "major loop" of the hysteresis.

Figure 3 shows a typical plot of the time evolution of the hysteresis in the $B^{i}$ versus $H^{i}$ plane for two different amplitude sinusoidal time functions, $H^{i}(t)$, satisfying the initial conditions $B^{i}(0)=0$ and $H^{i}(0)=0$. Note that if $H^{i}(0), B^{i}(0)$ lies inside the major loop then the evolution $H^{i}(t), B^{i}(t)$ always remains inside the major loop for all $t \geq 0$.

The meaning of the two hysteresis constants $H_{c}, H_{r}$ that appear in the above development is clarified by referring to the "major loop" as in Figure 3. It is sufficient to consider only the equality condition for the S-shaped major loop that corresponds to increasing values of $H^{i}$. The coercivity value satisfies:

$$
H^{i}=H_{c} \Rightarrow B^{i}=0
$$

and the remanence value satisfies:

$$
H_{r}=\cot \left(\frac{\pi B_{r}}{2 B_{m}}\right) H_{c}
$$

where 


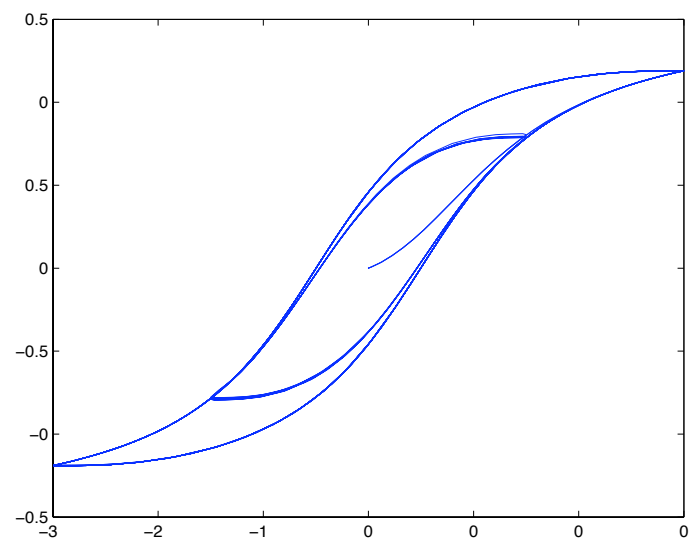

Figure 3. Typical hysteresis loops

$$
H^{i}=0 \Rightarrow B^{i}=-B_{r}
$$

That is, $H_{c}$ is the value for which this part of the major loop crosses the $B^{i}=0$ axis and $H_{r}$ is the value on the major loop for which $B^{i}=B_{r}$.

This model of magnetic hysteresis is similar to but slightly different from the Duhem models of frictioninduced hysteresis described in. ${ }^{11}$ It can be shown the hysteresis model used here is in fact rate-independent according to the definition in. ${ }^{11}$

The magnetic torque on the RAX nanosatellite due to the presence of hysteresis rod $i$, expressed in the body-fixed frame, is

$$
M^{i}=B^{i} V_{h} b_{i} \times R_{E}^{B} H_{E}, i=1,2,
$$

where $V_{h}$ is the volume of each hysteresis rod. The direction of the torque produced by hysteresis rod $i$ is orthogonal to the longitudinal axis of that hysteresis rod and to the direction of the Earth's magnetic field at the RAX location.

\section{F. Summary of Attitude Equations}

We now summarize the prior development to obtain a complete mathematical description of the magnetic attitude control system for the RAX attitude dynamics. The Euler equations are given by

$$
J \dot{\omega}+\omega \times J \omega=M_{p}+M^{1}+M^{2}
$$

and the attitude kinematics equations are given by

$$
\dot{R_{I}^{B}}+S(\omega) R_{I}^{B}=0
$$

The magnetic field induced in hysteresis rod 1 satisfies: if $\dot{H}^{1} \geq 0$, then

$$
\dot{B}^{1}=\frac{2 B_{m}}{H_{r} \pi}\left(\frac{\left(H^{1}+H_{c}\right) \cos \left(\frac{\pi B^{1}}{2 B_{m}}\right)-H_{r} \sin \left(\frac{\pi B^{1}}{2 B_{m}}\right)}{2 H_{c}}\right)^{2} \dot{H}^{1}
$$

while if $\dot{H}^{1}<0$, then 


$$
\dot{B}^{1}=\frac{2 B_{m}}{H_{r} \pi}\left(\frac{\left(H_{c}-H^{1}\right) \cos \left(\frac{\pi B^{1}}{2 B_{m}}\right)+H_{r} \sin \left(\frac{\pi B^{1}}{2 B_{m}}\right)}{2 H_{c}}\right)^{2} \dot{H}^{1}
$$

where

$$
\begin{gathered}
H^{1}=b_{1}^{T} R_{I}^{B}\left(R_{I}^{E}\right)^{T} H_{E} \\
\dot{H}^{1}=b_{1}^{T}\left(-S(\omega) R_{I}^{B}\left(R_{I}^{E}\right)^{T}+R_{I}^{B}\left(\dot{R}_{I}^{E}\right)^{T}\right) H_{E}+b_{1}^{T} R_{I}^{B}\left(R_{I}^{E}\right)^{T} \dot{H}_{E} .
\end{gathered}
$$

The magnetic field induced in hysteresis rod 2 satisfies: if $\dot{H}^{2} \geq 0$, then

$$
\dot{B^{2}}=\frac{2 B_{m}}{H_{r} \pi}\left(\frac{\left(H^{2}+H_{c}\right) \cos \left(\frac{\pi B^{2}}{2 B_{m}}\right)-H_{r} \sin \left(\frac{\pi B^{2}}{2 B_{m}}\right)}{2 H_{c}}\right)^{2} \dot{H}^{2}
$$

while if $\dot{H}^{2}<0$, then

$$
\dot{B^{2}}=\frac{2 B_{m}}{H_{r} \pi}\left(\frac{\left(H_{c}-H^{2}\right) \cos \left(\frac{\pi B^{2}}{2 B_{m}}\right)+H_{r} \sin \left(\frac{\pi B^{2}}{2 B_{m}}\right)}{2 H_{c}}\right)^{2} \dot{H}^{2}
$$

where

$$
\begin{gathered}
H^{2}=b_{2}^{T} R_{I}^{B}\left(R_{I}^{E}\right)^{T} H_{E} \\
\dot{H}^{2}=b_{2}^{T}\left(-S(\omega) R_{I}^{B}\left(R_{I}^{E}\right)^{T}+R_{I}^{B}\left(\dot{R}_{I}^{E}\right)^{T}\right) H_{E}+b_{2}^{T} R_{I}^{B}\left(R_{I}^{E}\right)^{T} \dot{H}_{E} .
\end{gathered}
$$

The magnetic torques on the RAX are given by

$$
\begin{aligned}
& M_{p}=B_{p} V_{p} b_{3} \times R_{I}^{B}\left(R_{I}^{E}\right)^{T} H_{E}, \\
& M^{1}=B^{1} V_{h} b_{1} \times R_{I}^{B}\left(R_{I}^{E}\right)^{T} H_{E}, \\
& M^{2}=B^{2} V_{h} b_{2} \times R_{I}^{B}\left(R_{I}^{E}\right)^{T} H_{E} .
\end{aligned}
$$

The Earth's magnetic field vector $H_{E}$ is time varying and is obtained from the IGRF model and depends on specific RAX orbital data. The time varying rotation matrix $R_{I}^{E}$ is

$$
R_{I}^{E}=\left[\begin{array}{ccc}
\cos \omega_{0} t & -\sin \omega_{0} t & 0 \\
\sin \omega_{0} t & \cos \omega_{0} t & 0 \\
0 & 0 & 1
\end{array}\right]
$$

and its time derivative $\dot{R}_{I}^{E}$ is

$$
\dot{R}_{I}^{E}=\left[\begin{array}{ccc}
-\omega_{0} \sin \omega_{0} t & -\omega_{0} \cos \omega_{0} t & 0 \\
\omega_{0} \cos \omega_{0} t & -\omega_{0} \sin \omega_{0} t & 0 \\
0 & 0 & 0
\end{array}\right]
$$

One useful performance measure for the magnetic attitude control system of the RAX nanosatellite is the angle between the longitudinal axis of the permanent magnet (the third principal axis of the RAX nanosatellite) and the direction of the Earth's magnetic field at the RAX location, expressed in the body-fixed frame. This angle can be written as 


$$
\theta=\cos ^{-1}\left(\frac{b_{3}^{T} R_{I}^{B}\left(R_{I}^{E}\right)^{T} H_{E}}{H_{E}}\right) .
$$

We refer to this angle as the attitude angle error, since if the longitudinal axis of the permanent magnet (the third principal axis of the RAX nanosatellite) is exactly aligned with the direction of the Earth's magnetic field at the location of the RAX, then this angle is zero. In analytical terms, the attitude angle is zero if the first two principal axes (the axes of the two hysteresis rods) of the RAX are orthogonal to the local direction of the Earth's magnetic field, that is $H^{1}=0, H^{2}=0$, and $\theta=0$ if:

$$
R_{I}^{B}=R_{I}^{E} \exp \left(\frac{-S\left(H_{E} \times b_{3}\right)}{H_{E}}\right) .
$$

\section{G. Initial Value Problems for the RAX Attitude Dynamics}

The above equations constitute a complete set of differential equations that describes the attitude dynamics of the RAX nanosatellite.

If initial values for RAX's attitude and angular velocity and the initial values of the magnetic field in the two hysteresis rods are specified, then a well-posed initial value problem is defined which can be numerically integrated to obtain the resulting attitude and angular velocity time responses. It should be mentioned that the initial values must satisfy the inequalities associated with the two hysteresis rods; in such cases, the resulting hysteresis inequalities remain valid and the complete attitude dynamics model for the RAX is physically meaningful.

This initial value problem is nonlinear, due to the specific form of the Euler and attitude kinematics equations and the fact that the configurations, namely the rotation matrices, do not define a linear space; the hysteresis rods are also fundamentally nonlinear. The problem is also time-varying, due to the fact that the Earth's magnetic field varies in magnitude and direction as RAX moves along its orbit.

\section{Analysis of the RAX Attitude Dynamics}

The fact that the equations for the RAX attitude dynamics are both nonlinear and time-varying makes formal analysis of the RAX attitude dynamics difficult. Several results and insights are presented based on theoretical analysis of the above model for the RAX attitude dynamics.

\section{A. RAX Attitude Dynamics in an Equatorial Circular Orbit}

Consider the case that the RAX nanosatellite is in an ideal equatorial circular orbit. Ideally, this implies that the Earth's magnetic field is constant in magnitude and direction along the RAX orbit. Several theoretical results for this case are summarized.

\section{RAX Relative Equilibrium Solutions}

It can be shown that there are exactly two classes of relative equilibrium solutions, each of which corresponds to identically zero attitude angle error. One class of relative equilibrium solutions can be described in mathematical terms, using Rodrigues theorem, ${ }^{3}$ as follows:

$$
\begin{gathered}
R_{I}^{B}=\exp \left(-S\left(b_{3}\right) \Omega t\right)=I_{3 \times 3} \cos (\Omega t)+b_{3}\left(b_{3}\right)^{T}(1-\cos (\Omega t))-S\left(b_{3}\right) \sin (\Omega t), \\
\omega=\Omega b_{3}, \\
B^{1}=B^{2}=0,
\end{gathered}
$$

where $\Omega$ is an arbitrary real number representing the scalar magnitude of the angular velocity of the RAX about its third principal axis. These relative equilibrium solutions occur when the direction from the south 
pole to the north pole of the permanent magnet is exactly aligned with the direction of magnetic north of the Earth's magnetic field, and the angular velocity of the RAX is along this direction with scalar magnitude $\Omega$. In any such relative equilibrium, the hysteresis rods remain orthogonal to the direction of the Earth's magnetic field, so that the magnetizing field along the hysteresis rods satisfies $H^{1}=H^{2}=0$.

The other class of relative equilibrium solutions can be described, again using Rodrigues theorem, in mathematical terms as follows:

$$
\begin{gathered}
R_{I}^{B}=\exp \left(S\left(b_{3}\right) \Omega t\right)=I_{3 \times 3} \cos (\Omega t)+b_{3}\left(b_{3}\right)^{T}(1-\cos (\Omega t))+S\left(b_{3}\right) \sin (\Omega t), \\
\omega=-\Omega b_{3}, \\
B^{1}=B^{2}=0,
\end{gathered}
$$

where $\Omega$ is an arbitrary real number representing the scalar magnitude of the angular velocity of the RAX about its third principal axis. These relative equilibrium solutions occur when the direction from the north pole to the south pole of the permanent magnet is exactly aligned with the direction of magnetic north of the Earth's magnetic field, and the angular velocity of the RAX is along this direction with scalar magnitude $\Omega$. In any such relative equilibrium, the hysteresis rods remain orthogonal to the direction of the Earth's magnetic field, so that the magnetizing field along the hysteresis rods satisfies $H^{1}=H^{2}=0$.

Note that the pointing directions of the RAX nanosatellite corresponding to these two classes of relative equilibrium solutions differ by a rotation of $\pi$ radians.

\section{Stability of RAX Relative Equilibrium Solutions}

We now summarize (without proof) certain stability properties of these relative equilibrium solutions. Each of the first class of relative equilibrium solutions is almost globally asymptotically stable. This implies that almost all solutions have the following properties: the angle error tends asymptotically to zero, that is:

$$
\lim _{t \rightarrow \infty} \theta(t)=0
$$

the first two components of the angular velocity tend asymptotically to zero and the third component of the angular velocity tends asymptotically to some constant value $\Omega$, that is:

$$
\lim _{t \rightarrow \infty} \omega_{1}=\lim _{t \rightarrow \infty} \omega_{2}=0, \lim _{t \rightarrow \infty} \omega_{3}=\Omega,
$$

and the magnetic flux densities induced in the hysteresis rods tend asymptotically to zero, that is:

$$
\lim _{t \rightarrow \infty} B^{1}=\lim _{t \rightarrow \infty} B^{2}=0 .
$$

Each of the second class of relative equilibrium solutions is unstable. Excluding only those solutions that asymptotically approach one of the unstable relative equilibrium solutions, all other solutions approach one of the first class of relative equilibrium solutions, as described above. Although magnetic effects and gravitational effects are physcially different, the methods presented in ${ }^{1}$ for a $3 \mathrm{D}$ pendulum in a gravitational field can be extended to formalize the stability results stated here.

\section{B. Analogy between RAX Attitude Dynamics and Dynamics of a Compass Needle}

It is perhaps helpful to draw an analogy between the 3D rotational motion of the RAX nanosatellite and the 1D rotational motion of a typical compass needle assuming a constant Earth magnetic field. The first class of RAX relative equilibrium solutions is analogous to the north pole of a compass needle pointing directly towards geomagnetic north; assuming there is some dissipation or damping in the compass needle, perturbations of the compass needle from geomagnetic north eventually decay and the compass needle asymptotically approaches geomagnetic north. On the other hand, the second class of relative equilibrium solutions is analogous to the south pole of the compass needle pointing directly towards geomagnetic north. Although this is an equilibrium for the compass needle, ideally, any small perturbation will cause the compass needle to 
rotate so that the north pole of the compass needle asymptotically approaches geomagnetic north. The additional complexity of the rotational motion of the RAX nanosatellite is primarily due to the complexity of rigid body rotations in $3 \mathrm{D}$ and the fact that the magnetic control system stabilizes the pointing direction of the third principal axis but does not control the RAX angular velocity about that principal axis.

\section{RAX Attitude Dynamics in a non-Equatorial Circular Orbit}

Equatorial, circular orbits are not of immediate interest, since near-polar orbits are planned for upcoming RAX missions. Nevertheless, the analytical results described above provide useful insight into the complex attitude dynamics of the RAX even in non-equatorial orbits.

Along non-equatorial, circular RAX orbits, there are no equilibrium or relative equilibrium solutions. The Earth's magnetic field varies in a complicated way along an orbit; it is often approximated as periodic or nearly-periodic with primary frequency content consisting of the orbital frequency and twice the orbital frequency; that is, along one orbit, the direction of the magnetic field can undergo approximately two revolutions. The Earth's magnetic field varies much more slowly than the rotational motion of the RAX. This time scale separation implies that the above results for the RAX attitude dynamics, assuming a constant Earth magnetic field, are relevant as an approximation to the RAX attitude dynamics in a non-equatorial orbit. We do not give formal statements, but we do summarize the nature of the approximation.

In a non-equatorial circular orbit, there is class of "steady state" RAX attitude motions that consists of a combination of periodic or nearly-periodic variations in the direction of the third principal axis of the RAX and nearly constant spin rotation about that axis. Such "steady state" motions are analogous to the relative equilibrium solutions in the ideal case of constant Earth magnetic field. The amplitude of these steady state motions is small and depends on the time scale separation mentioned above. In addition, these steady state motions have an almost global asymptotic stability property: almost all RAX attitude motions asymptotically approach one of the steady state attitude motions. These statements are heuristic and would require considerable work to formalize.

The difficulty of analyzing such complex RAX attitude motions provides motivation for reliance on computer simulations of the attitude dynamics of the magnetically controlled RAX nanosatellite in non-equatorial circular orbits.

\section{Attitude Dynamics Simulation Results}

We describe a computational approach to solving initial value problems associated with the attitude dynamics of the RAX nanosatellite based on the model previously introduced. We describe the deficiencies in using standard numerical integration algorithms, and we summarize the Lie group variational integrator algorithm used in the simulations and its computational properties. A select set of simulation results are summarized.

\section{A. Numerical integration algorithms and the Lie group variational integrator}

If the RAX nanosatellite were not to include hysteresis rods, and the Earth's magnetic field were assumed to have constant magnitude and direction, analytical methods could be used to demonstrate that the total energy, namely the kinetic energy plus the magnetic potential energy, should be conserved. In the case of interest here where there is a slow variation of the Earth's magnetic field along the RAX orbit, the total energy is not exactly conserved. Our expectation is that the energy dissipation rate due to the hysteresis rods is very small, so that many orbits may be required before the attitude reaches some steady state motion.

It is well known that standard numerical integration algorithms, such as Runge Kutta algorithms, do not exactly preserve theoretical energy properties, especially over long time periods, since they tend to introduce numerical dissipation. Moreover, such algorithms, when applied to the RAX attitude model, do not preserve the orthogonality of the computed rotation matrices that characterize the RAX attitude. Both of these factors imply that numerical errors in simulations using such algorithms will increase significantly after several RAX orbits, thereby invalidating the attitude responses obtained. 
Recently, numerical integration algorithms have been developed for Hamiltonian systems defined on configurations with a Lie group structure, that are guaranteed to have good energy preservation properties and to maintain the Lie group structure, even over long time periods. ${ }^{6,7}$ A specific Lie group variational integrator for rigid body attitude models has been developed; it is easily modified to incorporate the dissipation due to the RAX hysteresis rods. This is the numerical algorithm that is used to obtain the subsequent simulations results. This Lie group variational integrator provides confidence that the computed simulation results for the RAX attitude dynamics are accurate, even over many orbits.

\section{B. Simulation Results}

To verify our model, Matlab was used with the Lie Group variational integrator code to numerically integrate the differential equations of motions for the attitude kinematics and attitude dynamics. The Matlab code also incorporates a simple forward Euler integration scheme to update the induced magnetic flux density in the two hysteresis rods. In both algorithms, a fixed and constant step size is selected. A circular orbit is assumed and the IGRF model for the Earth magnetic field is incorporated into the computation of the magnetic torques due to the permanent magnet and the hysteresis rods. Simulation results are presented for the RAX is a circular orbit at several different inclinations.

\section{RAX Simulation Assumptions}

The orbital specifications for the RAX nanosatellite are as follows. The RAX is in a circular orbit at an altitude of $650.0 \mathrm{~km}$. The planned inclination angle of the orbital plane of the RAX is $72.0^{\circ}$; simulation results are presented for this inclination angle and for inclination angle of $0.0^{\circ}$. The moment of inertia matrix of the RAX nanosatellite is $J=\operatorname{diag}(2.91058 ; 2.91058 ; 0.59261) \times 10^{-2} \mathrm{~kg}-\mathrm{m}^{2}$, reflecting the fact that the third principal axis of the RAX is an axis of symmetry. The permanent magnet is characterized by the magnetic flux density value $B_{p}=1.28$ Teslas and by the volume $V_{p}=3.0137 \times 10^{-6} \mathrm{~m}^{3}$. The two hysteresis rods are assumed to be identical, with volume $V_{h}=7.15 \times 10^{-8} \mathrm{~m}^{3}$ and hysteresis constants $B_{m}=0.73$ Teslas, $H_{c}=1.59 \mathrm{~A} / \mathrm{m}$, and $H_{r}=1.696 \mathrm{~A} / \mathrm{m}$. The Earth's magnetic field data is obtained from the standard International Geomagnetic Reference Field (IGRF) ${ }^{13}$ that provides numerical values for the magnetic field vector in Earth-centered Earth-fixed frame at each point along the RAX orbit. The RAX orbit position vector should be expressed in the Earth-centered Earth-fixed frame. The constant step size is selected as $0.05 \mathrm{sec}$ and the total simulation time is for six orbital periods.

The basic Matlab code used in the simulation (for orbital inclination angle $72.0^{\circ}$ ) is listed in the Appendix. There is a main $\mathrm{m}$-file that numerically integrates the attitude kinematics and attitude dynamics using the Lie group variational integrator. The magnetic moments and the hysteresis variables are updated in moment.m; this m-file calls IGRF.m and two m-files that transform the inertial frame to the Earth-fixed frame. The main m-file calls output.m to develop suitable plots for the RAX rotational motion.

Four different sets of simulation results are provided. Scenario 1 assumes that the RAX is in a zero degree inclination circular orbit; it is defined by initial conditions that correspond to an ideal RAX deployment for which the initial angle error is zero and the initial angular velocity is zero. Scenario 2 assumes that the RAX is in a zero degree inclination circular orbit; it is defined by initial conditions that correspond to an inaccurate RAX deployment for which the initial angle error is zero but the initial angular velocity is large. Scenario 3 assumes that the RAX is in a 72 degree inclination circular orbit; it is defined by initial conditions that correspond to an ideal RAX deployment for which the initial angle error is zero and the initial angular velocity is zero. Scenario 4 assumes that the RAX is in a 72 degree inclination circular orbit; it is defined by initial conditions that correspond to an inaccurate RAX deployment for which the initial angle error is zero but the initial angular velocity is large. In all scenarios, the magnetic flux density in each hysteresis rod is initialized as zero. Simulation results are first presented and then the significance of the results is discussed. 


\section{Simulation Scenario 1}

The RAX attitude motion is simulated for an orbital inclination of 0 degrees. The initial attitude of the RAX, viewed as an initial rotation matrix that relates the body frame and the inertial frame, is

$$
R(0)=\left[\begin{array}{ccc}
0.9990 & -0.0076 & 0.0448 \\
0.0076 & 0.9437 & 0.3308 \\
-0.0448 & -0.3308 & 0.9426
\end{array}\right]
$$

and the initial angular velocity of the RAX is

$$
\omega(0)=\left[\begin{array}{lll}
0 & 0 & 0
\end{array}\right] \mathrm{rad} / \mathrm{sec}
$$

The initial value for the hysteresis rods are $B^{1}(0)=0, B^{2}(0)=0$. The initial conditions correspond to an ideal RAX deployment for which the initial attitude error is zero, that is eqn.(33) is initially satisfied.

Simulation results for the pointing error versus time, for the components of the angular velocity vector versus time, and for the memory in the hysteresis rods are given in the following plots.

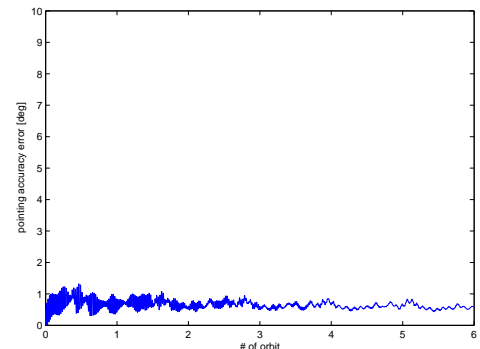

(a) Pointing Error

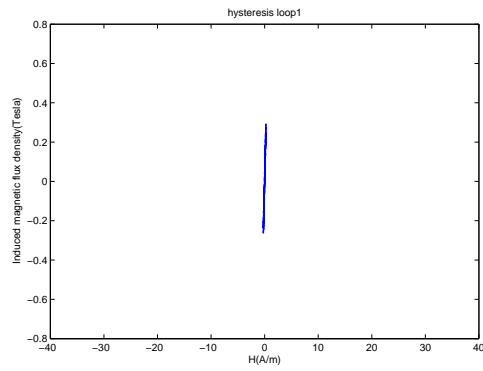

(c) Hysteresis rod1

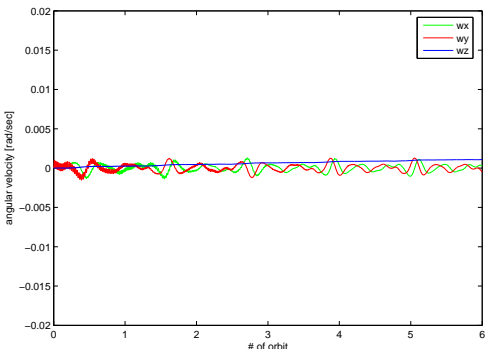

(b) Angular Velocity

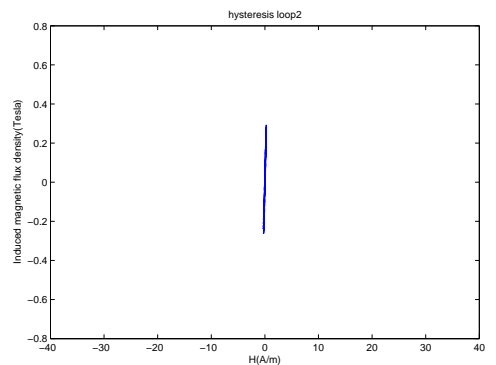

(d) Hysteresis rod2

Figure 4. Simulation for orbit inclination angle of 0.0 degrees

\section{Simulation Scenario 2}

The RAX attitude motion is simulated for an orbital inclination of 0 degrees. The initial attitude matrix of the RAX, viewed as an initial rotation matrix that relates the body frame and the inertial frame, is

$$
R(0)=\left[\begin{array}{ccc}
0.9990 & -0.0076 & 0.0448 \\
0.0076 & 0.9437 & 0.3308 \\
-0.0448 & -0.3308 & 0.9426
\end{array}\right]
$$

and the initial angular velocity of the RAX is

$$
\omega(0)=\left[\begin{array}{lll}
0.05 & 0.05 & 0.05
\end{array}\right] \mathrm{rad} / \mathrm{sec}
$$


The initial value for the hysteresis rods are $B^{1}(0)=0, B^{2}(0)=0$. The initial conditions correspond to an inaccurate RAX deployment for which the initial angular velocity is large, thereby leading to subsequent large pointing angle errors.

Simulation results for the pointing error versus time, for the components of the angular velocity vector versus time, and for the memory in the hysteresis rods are given in the following plots.

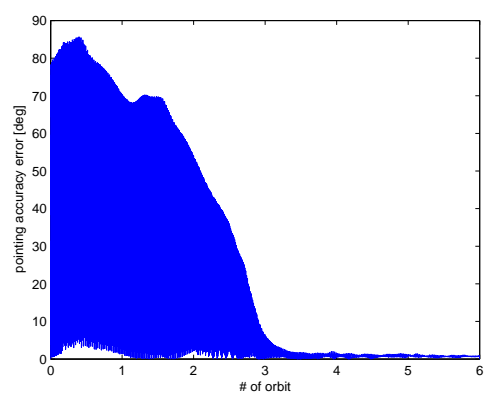

(a) Pointing Error

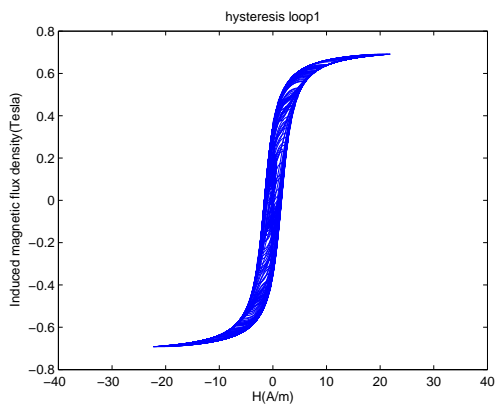

(c) Hysteresis rod1

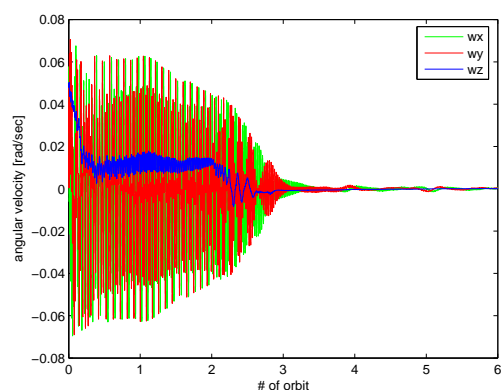

(b) Angular Velocity

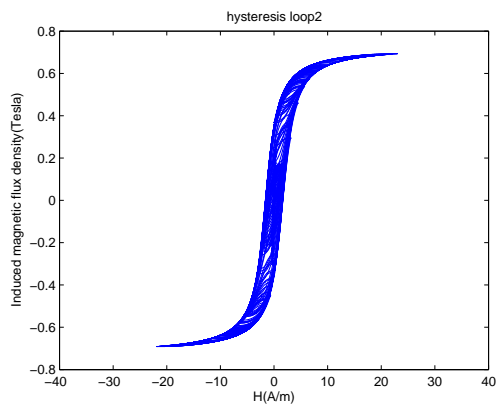

(d) Hysteresis rod2

Figure 5. Simulation for orbit inclination angle of 0.0 degrees

\section{Simulation Scenario 3}

The RAX attitude motion is simulated for an orbital inclination of 72 degrees. The initial attitude of the RAX, viewed as an initial rotation matrix that relates the body frame and the inertial frame, is

$$
R(0)=\left[\begin{array}{ccc}
0.9947 & -0.1008 & -0.0221 \\
0.0102 & 0.1172 & -0.9931 \\
0.1027 & 0.9880 & 0.1155
\end{array}\right]
$$

and the initial angular velocity of the RAX is

$$
\omega(0)=\left[\begin{array}{lll}
0.0 & 0.0 & 0.0
\end{array}\right] \mathrm{rad} / \mathrm{sec}
$$

The initial value for the hysteresis rods are $B^{1}(0)=0, B^{2}(0)=0$. The initial conditions correspond to an ideal RAX deployment for which the initial attitude error is zero, that is eqn.(33) is initially satisfied.

Simulation results for the pointing error versus time, for the components of the angular velocity vector versus time, and for the memory in the hysteresis rods are given in the following plots. 


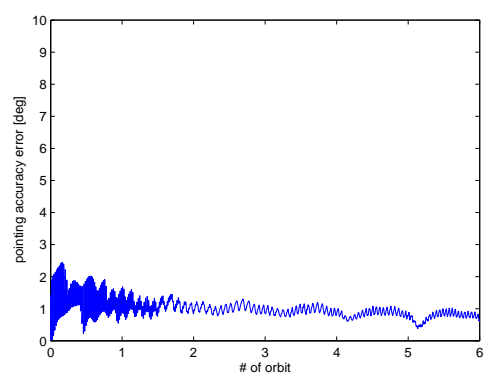

(a) Pointing Error

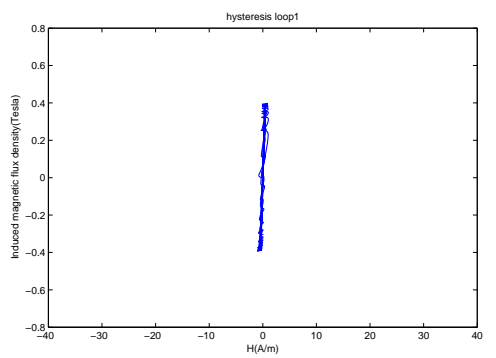

(c) Hysteresis rod1

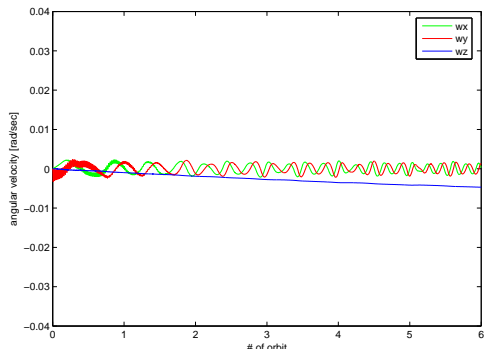

(b) Angular Velocity

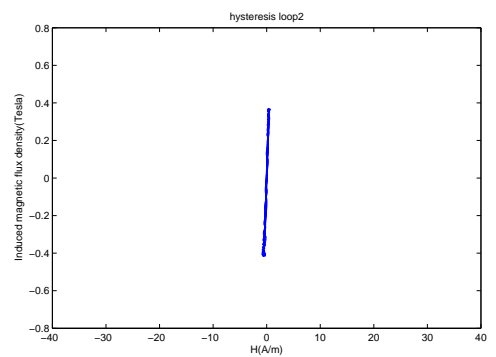

(d) Hysteresis rod2

Figure 6. Simulation for orbit inclination angle of 72.0 degrees

\section{Simulation Scenario 4}

The RAX attitude motion is simulated for an orbital inclination of 72 degrees. The initial attitude matrix of the RAX, viewed as an initial rotation matrix that relates the body frame and the inertial frame, is

$$
R(0)=\left[\begin{array}{ccc}
0.9947 & -0.1008 & -0.0221 \\
0.0102 & 0.1172 & -0.9931 \\
0.1027 & 0.9880 & 0.1155
\end{array}\right]
$$

and the initial angular velocity of the RAX is

$$
\omega(0)=\left[\begin{array}{lll}
0.05 & 0.05 & 0.05
\end{array}\right] \mathrm{rad} / \mathrm{sec}
$$

The initial value for the hysteresis rods are $B^{1}(0)=0, B^{2}(0)=0$. The initial conditions correspond to an inaccurate RAX deployment for which the initial angular velocity is large, thereby leading to subsequent large pointing angle errors.

Simulation results for the pointing error versus time, for the components of the angular velocity vector versus time, and for the memory in the hysteresis rods are given in the following plots.

\section{RAX Simulation Results}

These are only a small set of simulation results, but the following conclusions can be made on the basis of these results. The pointing accuracy of the RAX has the following properties.

- In Scenario 1, for which the RAX is in an equatorial orbit, the pointing error remains less than 1.5 degrees; this rotational motion essentially describes the best case pointing accuracy as the magnetic control systems causes the axis of symmetry of the RAX to track the slowing changing direction of the Earth's magnetic field. 


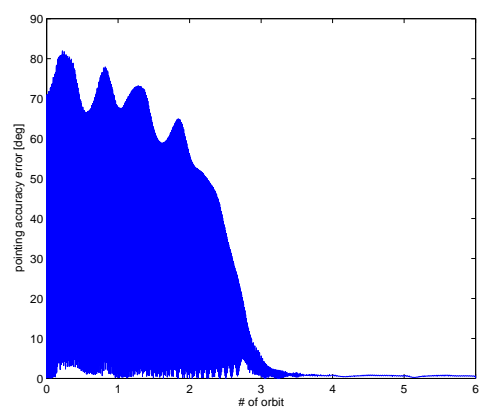

(a) Pointing Error

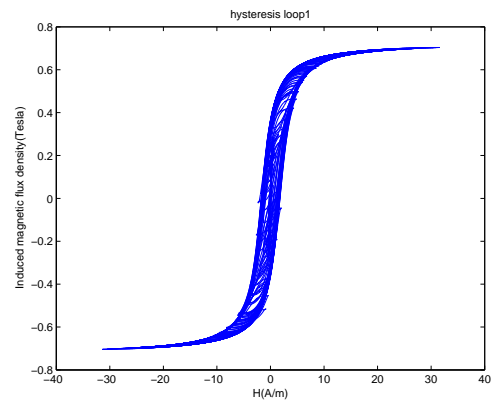

(c) Hysteresis rod1

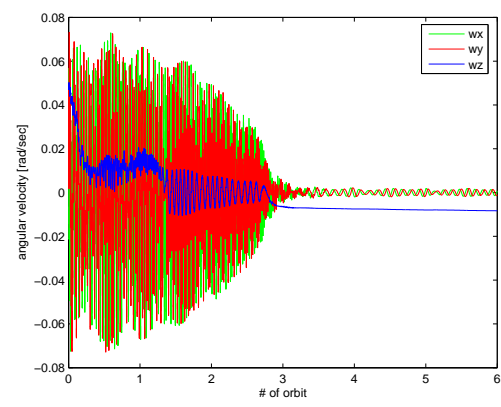

(b) Angular Velocity

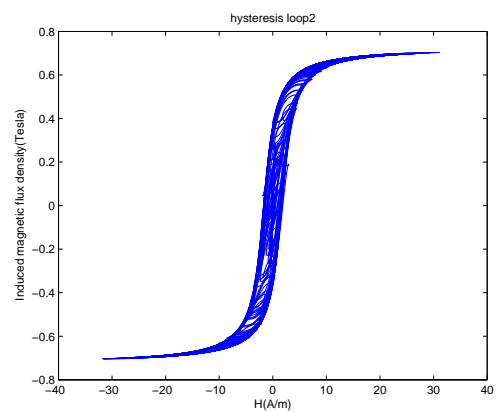

(d) Hysteresis rod2

Figure 7. Simulation for orbit inclination angle of 72.0 degrees

- In Scenario 2, for which the RAX is in an equatorial orbit, the maximum pointing error reaches about 80 degrees due to the large initial angular velocity of the RAX after deployment; as energy is dissipated by the hysteresis rods, the pointing error slowly is reduced over several orbits. After three orbits the attitude error is reduced to less than 5.0 degrees.

- These results hold for Scenarios 1 and 2 hold for an equatorial orbit where the variation in the direction of the Earth's magnetic field is smallest.

- In Scenario 3, for which the RAX has an orbit inclination angle of 72.0 degrees, the pointing error remains within 2.5 degrees. This rotational motion essentially describes the best case pointing accuracy as the magnetic control systems causes the axis of symmetry of the RAX to track the slowing changing direction of the Earth's magnetic field.

- In Scenario 4, for which the RAX has an orbit inclination angle of 72.0 degrees, the maximum pointing error reaches about 70 degrees but slowly decreases over several orbits.

- The results for Scenario 4 demonstrate somewhat slower energy dissipation than for Scenario 2, presumably due to the fact that the magnitude and direction of the Earth's magnetic field have large variations due to the inclination angle of the RAX orbit.

The hysteresis loops of the RAX have the following properties.

- In Scenarios 1 and 3 the induced magnetic fluxes remain small; there is little dissipation of energy required by the hysteresis rods in these scenarios.

- In Scenarios 2 and 4 the induced magnetic fluxes fill out the major hysteresis loops, indicating that there is substantial dissipation of energy required by the hysteresis rods in these scenarios.

It is difficult to draw general conclusions about this limited set of simulation results, but the results are consistent with the theoretical results mentioned previously. Much additional investigation is required to develop insight into the specific effects of deployment and the dependence on the orbital inclination angle, orbital radius, and physical properties of the RAX. 


\section{Conclusions}

This paper has presented a formal, integrated model for the attitude dynamics of the RAX nanosatellite. The model assumes RAX is in a low Earth orbit and is being acted upon by magnetic control torques due to a permanent magnet and two hysteresis rods embedded in the satellite. This model is globally defined in the sense that there are no singularities; as such, it fully captures the tumbling dynamics following deployment and the slow convergence towards some steady state rotational motion. Some formal results for the RAX attitude dynamics have been presented assuming a constant Earth magnetic field; theoretical implications for the RAX attitude dynamics in the case of arbitrary RAX circular orbits have been suggested. Typical simulations have been presented, based on the use of numerically accurate LGVI methods. These simulations provide a basis for the assessment of the strengths and weaknesses of this type of passive magnetic attitude control. The RAX attitude dynamics are dominated by the magnetic torques, which is why they have been the central feature of the presented results and simulations. The simulations do not include the effects of gravity gradient or aerodynamic torques; these additional torques can be included in the simulations without significant difficulties.

\section{Matlab Simulation M-files}

The prior simulation results were obtained using Matlab. The Matlab simulation code, as m-files, can be obtained on request from the authors.

\section{Acknowledgments}

The authors thank Professor J. Cutler, University of Michigan, for suggesting this passive attitude control problem and Professor T. Lee, Florida Institute of Technology, for his help with the Lie group variational integrator algorithm and its implementation in the simulation studies.

\section{References}

${ }^{1}$ N. A. Chaturvedi and N. H. McClamroch, Asymptotic Stabilization of the Hanging Equilibrium Manifold of the 3D Pendulum, International Journal of Robust and Nonlinear Control, Vol. 17, No. 6, 1435-1454, 2007.

${ }^{2}$ Flatley, T. W. and D. A. Henretty, A Magnetic Hysteresis Model, Paper 38, Proceedings of the Flight Mechanics/Estimation Theory Symposium, Greenbelt, MD, 1995.

${ }^{3}$ Hughes, P. C., Spacecraft Attitude Dynamics, Wiley, 1986.

${ }^{4} \mathrm{Kim}, \mathrm{K}$., Analysis of Hysteresis for Attitude Control of a Microsatellite, San Jose State University.

${ }^{5}$ R.R. Kumar, D.D. Mazanek, M.L. Heck, Simulation and Shuttle Hitchhiker Validation of Passive Satellite Aerostabilization, Journal of Spacecraft and Rockets, Vol. 32, No. 5, 1995.

${ }^{6}$ Lee, T., M. Leok, and N. H. McClamroch, Optimal Attitude Control of a Rigid Body using Geometrically Exact Computations on $\mathrm{SO}(3)$, Journal of Dynamical and Control Systems, Vol. 14, No. 4, 465-487, October, 2008.

${ }^{7}$ Lee, T., M. Leok, and N. H. McClamroch, Discrete Control Systems, Springer Encyclopedia of Complexity and System Science, 2009.

${ }^{8}$ Lee, T., M. Leok, and N. H. McClamroch, A Lie Group Variational Integrator for the Attitude Dynamics of a Rigid Body with Applications to the 3D Pendulum, Proceedings of the 2005 IEEE Conference on Control Applications, Toronto, Canada, 2005.

${ }^{9}$ Levesque, J.-F., Passive Magnetic Attitude Stabilization using Hysteresis Materials, SigMA-PU-006-UdeS.

${ }^{10}$ Menges, B.M., Guadiamos, C.A., and Lewis, E.K., Dynamic Modeling of Micro-Satellite Spartnik's Attitude, San Jose State University, Spartnik Satellite Project, Dec. 1996 SJSU.

${ }^{11}$ Padthe, A. K., B. Drincic, J. Oh, D. D. Rizos, S. D. Fassois, and D.S. Bernstein, Duhem Modeling of Friction-Induced Hysteresis, IEEE Control Systems Magazine, 90-107, October, 2008.

${ }^{12}$ Pais, D. and S. Jayaram, Satellite Passive Attitude Stabilization using Permanent Magnets: Dynamic Model and Simulation, AIAA paper.

${ }^{13}$ S. A. Rawashdeh and J. E. Lumpp, Nano-Satellite Passive Attitude Stabilization Systems Design by Oribal Environment Modeling and Simulation, AIAA Infotech, AIAA 2010-3413, Atlanta, GA, 2010.

${ }^{14}$ Roithmayr, C. M., Contributions of Spherical Harmonics to Magnetic and Gravitational Fields, NASA Technical Report TM-2004-213007,2004.

${ }^{15}$ Santoni, F. and M. Zelli, Passive Magnetic Attitude Stabilization of the UNISAT-4 Microsatellite, Acta Astronautica, 65, 2009.

${ }^{16}$ Whisnant, J. M., D.K. Anand, V.L. Pisacane and M. Sturmanis, Dynamic Modeling of Magnetic Hysteresis, Journal of Spacecraft and Rockets, Vol. 7, No. 6, 1970.

${ }^{17} \mathrm{http}: / /$ rax.engin.umich.edu 